\title{
A UNIQUE QUADRIFURCATION OF THE SCIATIC NERVE IN THE LOWER LEG
}

\author{
Afadhali D. Russa ${ }^{1}$ and Flora M. Fabian ${ }^{2}$
}

1. Department of Anatomy, School of Medicine, Muhimbili University of Health and Allied Sciences (MUHAS), Dar es Salaam, Tanzania.

2. Departments of Anatomy, College of Health Sciences, University of Dodoma, (UDOM), Dodoma, Tanzania.

Correspondence to Dr. Afadhali D. Russa, Department of Anatomy, School of Medicine, Muhimbili University of Health Sciences and Allied Sciences, P. O. Box 65001, Dares Salaam Email: adrussa@yahoo.com, drussa@muhas.ac.tz Tel: +255 755524 771.Fax: +255 222150465

\section{ABSTRACT}

Sciatic nerve is the largest nerve of the body supplying the entire posterior aspect of the lower limb. Taking its origin from the lumbosacral plexus, the nerve divides into its terminal branches at the superior angle of the popliteal fossa. Variant division patterns of the nerve especially those occurring in the thigh and the popliteal fossa are common. Divisions of the nerve occurring in the leg are rare. Even much rarer are terminations involving multiple branching patterns. Variations in the course and distribution of the sciatic nerve are of great importance in neurology, orthopedics, rehabilitation, anesthesia and many other clinical procedures. In the present study, we report a unique quadrifurcation termination of the sciatic nerve occurring deep in the distal half of the leg as observed during routine dissection by the medical students. Due to its variant muscular innervations, quadrifurcation and low divisions of the sciatic nerve may be of great importance in procedures such fracture management of the leg and foot, physiotherapy, limb amputation and in sports medicine. Embryologically, late separation of the common nerve sheath into individual terminal branches may lead to the lower division of the nerve.

KEY WORDS: Sciatic nerve, quadrifurcation, lower division, fracture

\section{INTRODUCTION}

Sciatic nerve, the largest nerve of the body, is formed by the anterior rami of L4 to S3 that converge at the inferior border of the piriformis muscle. It consists of two components almost independent of each other-the tibial and the common fibular (peroneal) nerves-that are loosely bound together in a connective tissue sheath. The tibial component is formed from the ventral branch of ventral rami of L4 to S3 spinal nerves. The common peroneal component is formed from the dorsal branches of ventral rami of L4 to S2 spinal nerves. The nerve passes through the inferior part of the greater sciatic foramen at the lower border of the piriformis muscles to enter the gluteal region and runs under cover of the gluteus maximus midway between the greater tronchanter of femur and ischial tuberosity. It passes posterior to the obturator internus, quadratus femoris and adductor magnus muscles and descends along the posterior aspect of the thigh supplying its supply muscles. The sciatic nerve supplies the skin of the foot, leg and foot muscles and all joints of the lower limb. The nerve divides into its terminal branches at the superior angle of the popliteal fossa (Standring 2005). Variant termination patterns of the nerve above the popliteal fossa are common (Saleh et al., 2001; Guvencer et al., 2009; Shewale et al., 2013)

Submitted $4^{\text {th }}$ October 2016, accepted May 2017. Russa AD, Fabian AF. A unique quadrifurcation of the sciatic nerve in the lower leg. Anatomy Journal of Africa. 2017. Vol 6 (2): 919 -923. 
and most occurs in the thigh (Ugrenovic et al., 2005, Prakash et al., 2010). These terminations in the thigh-often called high divisions-are implicated in several clinical conditions including the piriformis syndrome, sciatica, coccygodynia and muscular atrophy (Babinski et al., 2003). Lower sciatic nerve divisions are relatively less reported (Rajendiran and Manivasagam, 2015) and when they occur, may be associated with failures in nerve block (Vloka et al., 2001). Even much rarer are terminations involving multiple branching patterns. In the present study, we report a unique quadrifurcation termination of the sciatic nerve occurring deep in the distal half of the leg as observed during routine dissection by the medical students. We also review its possible clinical implications and embryological basis of variation.

\section{CASE REPORT}

During routine dissection classes of undergraduate medical students, we observed a lower division of the sciatic nerve in the posterior aspect of the right leg of an adult female cadaver of unknown age. The gluteal region and the posterior aspect of the thigh and leg of the cadaver were carefully dissected to expose the course and division of the sciatic nerve. The nerve emerged at the inferior border of the piriformis muscle, supplied the hamstring muscles (semimembranosus, semitendinosus and biceps femoris) on the posterior aspect of the thigh, and then passed through the popliteal fossa (Figure 1). The variation was unilateral involving the right limb whereas the left limb was normal.
The nerve divided into quadruple terminal branches (A, B, C, and D). Three of the four terminal branches were large and supplied muscles in the deep posterior aspect of the leg (flexor hallucis longus, tibialis posterior and flexor hallucis longus). The fourth smaller terminal branch passed deep to the tibialis posterior muscle, pierced the interosseous membrane to supply muscles of the posterior aspect of the leg. (Figure 2). The branching occurred $16 \mathrm{~cm}$ from the usual site of its termination-the superior angle of the popliteal fossa (Figure 3).

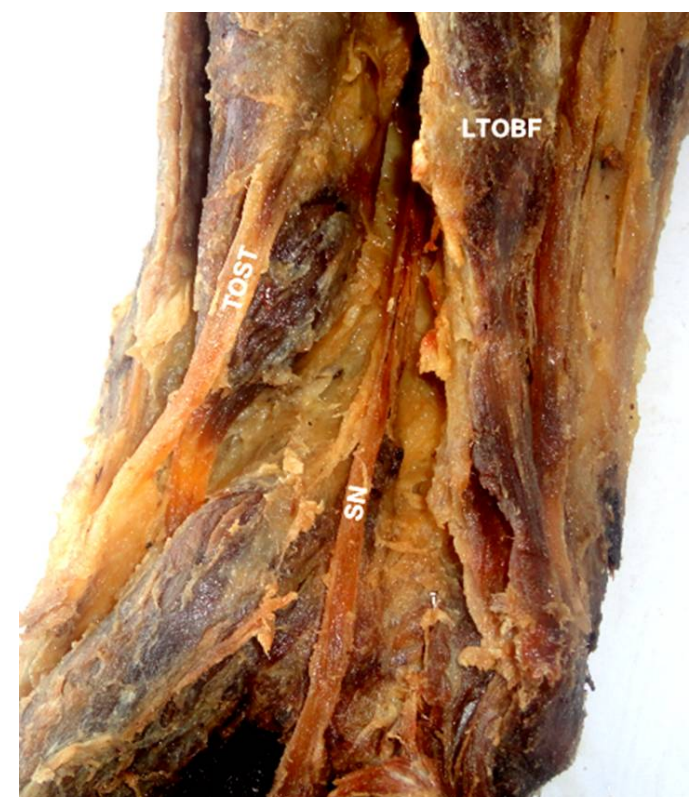

Figure 1: Sciatic nerve passing through the popliteal fossa between the tendon of semitendinosus and long head of biceps femoris. LTOB, long tendon of biceps femoris; SN, scistic nerve; TOST, tendon of semitendinosus. 


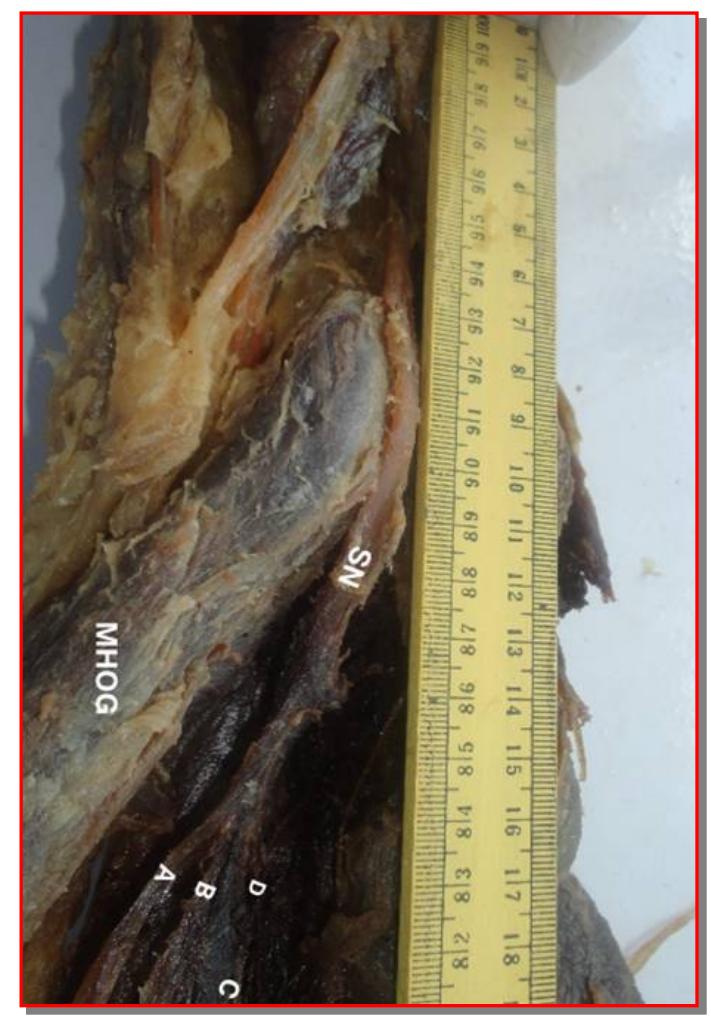

Figure 2: Sciatic nerve dividing into four terminal branches - A, B, C and D $16 \mathrm{~cm}$ from the superior angle of the popliteal fossa. MHOG, medial head of gastrocnemius muscle; SN, sciatic nerve

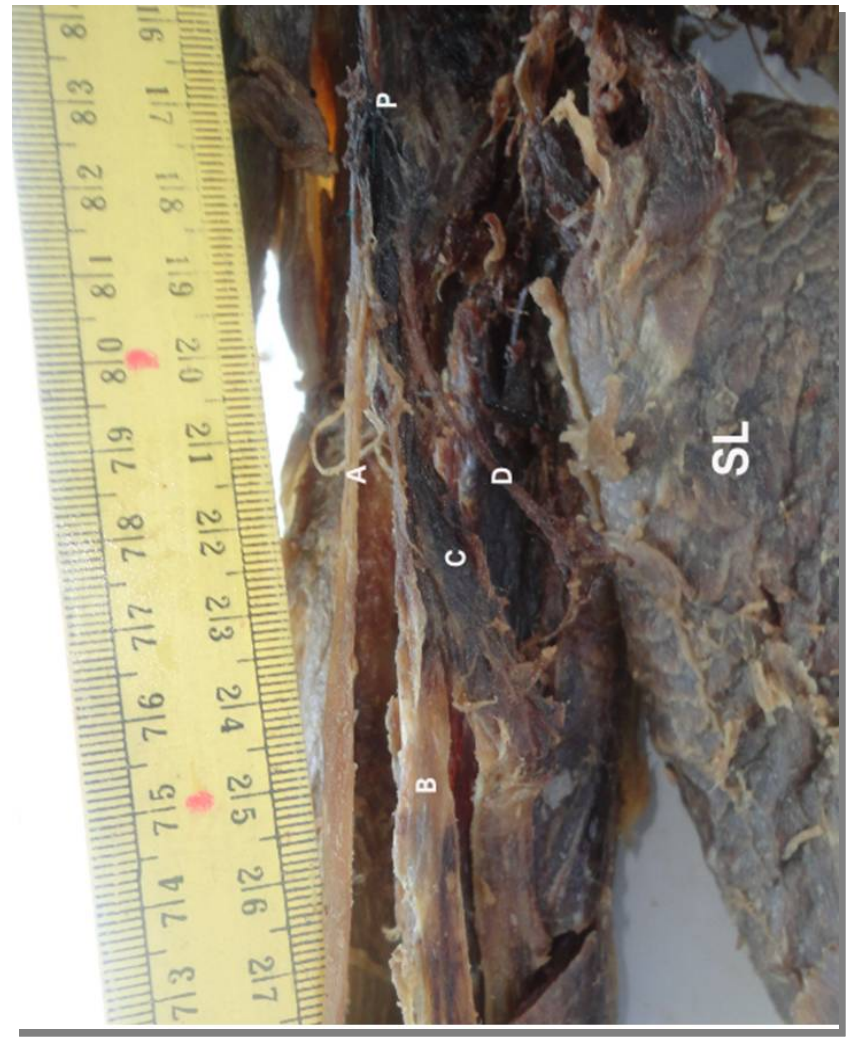

Figure 3: Sciatic nerve terminal branches (A, B, C and D) supplying posterior leg muscles. $P$, popliteal fossa (lower angle); SL, soleus muscles (reflected left)

\section{DISCUSSION}

Division of the sciatic nerve into its terminal branches may occur anywhere between the sacral plexus and the lower part of the popliteal fossa. High divisions of the sciatic nerve are the most encountered variations (Patel et al., 2011; Diogo et al., 2013; Shrivastana et al., 2014). Variations in the terminal branching patterns have also been reported (Sabnis, 2012; Saritha et al., 2012; Azejindu et al., 2013) and trifurcation is the most commonly encountered variation (Sawant, 2013). The present variation is unique in two aspects: the quadrifurcation and the lower division in the calf muscles of the leg. Variations in the course and distribution of the sciatic nerve are of great importance in neurology, orthopedics, rehabilitation, anesthesia (Rajendiran and Manivasagam, 2015) and many other clinical procedures. Due to its variant muscular innervations, quadrifurcation and low division reported in the present work may be of great importance in procedures such fracture management of the leg and foot, physiotherapy, limb amputation and in sports medicine.

Vloka et al. (2001) found that the division of the sciatic nerve occurs at variable levels 0-115 $\mathrm{mm}$ above the popliteal crease whereas Saleh et al. (2009) found that the division occurred 
50-180 $\mathrm{mm}$ above the crease. Ugrenovic et al (2005) showed that the sciatic nerve separated into the tibial and the common peroneal (fibular) nerves in $72.5 \%$ of the cases, whereas in the remainder; the sciatic nerve division was the high type $(27.5 \%)$ occurring either in the posterior femoral or in the gluteal region). Prakash et al. (2010) reported an incidence of $40.7 \%$ of division of the nerve occurring in the lower compartment of the thigh, $34.9 \%$ in the popliteal fossa and $16.3 \%$ of divisions observed proximal to the entrance into the gluteal region. Unlike the findings of the present study in which the termination was found $16 \mathrm{~cm}$ below the popliteal fossa, the distances of sciatic nerve termination reported in the above three studies are all either above or within the popliteal fossa-the landmark reference point. Embryologically, the sciatic nerve is formed when the dorsal larger component of the sacral plexus destined for posterior musculature and the smaller ventral component of the sacral plexus (anterior musculature) move downward close together within a common nerve sheath (Standring, 2007). As the limb elongate limb, late separation of the common nerve sheath into individual terminal branches in the popliteal fossa may lead to the division taking place in the leg.

Competing Interests: The authors declare that they have no financial or other competing interests.

Acknowledgement: Authors are grateful to the first-year medical students who reported the case for detailed studies and to the support staff of the Department of Anatomy and Histology, the University of Dodoma who prosected and presented the tissues.

\section{REFERENCES}

1. Babinski MA, Machado FA, Costa WS. 2003. A rare variation in the high division of the sciatic nerve surrounding the superior gemellus muscle. Eur J of Morphol, 41: 4142.

2. Diogo P, Diogo C, Pires B et al. 2013. Maria Alexandre, Furtado Andrea, Bilhim Tiago, angelica Almeida, Maria Goyri-O'Neill Joao. Sciatic nerve high division: two different anatomical variants. Acta Medica Portuguesa. 26: 208-111.

3. Ezejindu DN, Chinweife Kc, Nwajagu GI, Nzotta NO. 2013.The variations in the bifurcation of the sciatic nerve.G.J.B.A.H.S. 2: 20-23.

4. Guvencer M, Iyem C, Akyer P et al. 2009. Variations in the high division of the Sciatic nerve and relationship between the Sciatic nerve and the piriformis. Turk Neurosurg 68: 256-259.

5. Natsik K, Tatlis T, Konstantidinis GA et al. 2014. Anatomical variations between the Sciatic nerve and the piriformis muscle: a contribution to surgical anatomy in piriformis syndrome. Surg Radiol 36: $273-80$.

6. Nayak S. 2006. An unusual case of trifurcation of the Sciatic nerve. Neuroanat 5: 6-7.

7. Patel S, Vora MR, Zalawadia A, Rathod SP. 2011. A variation in the high division of the sciatic nerve and its relation with the piriformis muscle. Nat J Med Res 1: 27-30.

8. Prakash, Bradwaj AK, Devi MN et al. 2010. Singh G. Sciatic nerve division: A cadaver study in the Indian population and the review of the literature. Singapore Med J 51: 721-723.

9. Rajendiran R, Manivasagam M, 2015. Low level division of the sciatic Nerve and its clinical significance - case report. Brunei Darussalam J Health 6: 35-38.

10. Sabnis AS. 2012. Anatomical variations of Sciatic nerve bifurcation in human cadavers. J Clin Res Letters. 3: 46-48.

11. Saleh HA, El-fark MM, Abdel-Hamid GA. 2009. Anatomical variation of sciatic nerve division in the popliteal fossa and its implication in popliteal nerve blockade. Folia Morphol 68: 256-257.

12. Saritha S, Praveen Kumar M, Supriya G. 2012. Anatomical Variations in the Bifurcation of the Sciatic Nerve, A Cadaveric Study and its Clinical Implications. Anat Physiol 2: 111. 
13. Sawant SP, Shaikh ST, Lele SD et al. 2013. A case report on bilateral trifurcation of the sciatic nerve and variant formation of sural nerve. Intl J Res and Review Pharm and Applied Sc 3: 118124.

14. Shewale AD, Karambelkar RR, Umarji BN. 2013. Study of variations in the divisions, course and termination of the sciatic nerve. JKIMSU 2: 62-68.

15. Shrivastana T, Garg L, Mishra BK, Chhara N. 2014. High division of sciatic nerve. Int. J Res Med Sci., 2: 686-688.

16. Standring S. 2005. Gray's Anatomy, 39th Ed, London: Elsevier Churchill Livingstone. pp 13641458.

17. Ugrenovic $S$, Jovanovic I, Krstic $V$ et al. 2005. The level of the Sciatic nerve division and its relations to the piriformis muscle. Vojnosanit Pregl 62: 45-49.

18. Vloka JD, Hadzic A, April E, Thys DM. 2001. The division of the sciatic nerve in the popliteal fossa: anatomical implications for popliteal nerve blockade. Anesth Analg 92: 215-217. 